\title{
Short-period solar cycle signals in the ionosphere observed by FORMOSAT-3/COSMIC
}

\author{
Mao-Chang Liang, ${ }^{1,2}$ King-Fai Li, ${ }^{3}$ Run-Lie Shia, ${ }^{3}$ and Yuk L. Yung ${ }^{3}$ \\ Received 23 April 2008; revised 30 June 2008; accepted 11 July 2008; published 15 August 2008.
}

[1] We analyze 2 years of the FORMOSAT-3/COSMIC GPS radio occultation data to study the response of the Earth's ionosphere to the solar rotation (27-day) induced solar flux variations. Here we report electron density variations in the ionosphere $(\sim 100-500 \mathrm{~km})$ associated with the 27-day solar cycle. The peak-to-peak variation in electron density at low latitudes in the F2 region is about $\sim 10^{4}-10^{5}$ electrons $\mathrm{cm}^{-3}$ or $20-40 \%$, and can be as high as $60 \%$ depending on altitude, latitude, and season. The half and double periods of the 27-day are also observed at an amplitude comparable to that of the 27-day. The results place useful constraints for modeling chemical and dynamical processes in the ionosphere. Citation: Liang, M.-C., K.-F. Li, R.-L. Shia, and Y. L. Yung (2008), Short-period solar cycle signals in the ionosphere observed by FORMOSAT3/COSMIC, Geophys. Res. Lett., 35, L15818, doi:10.1029/ 2008 GL034433.

\section{Introduction}

[2] The GPS radio occultation instruments onboard the Constellation Observing System for Meteorology, Ionosphere and Climate (COSMIC) offer an opportunity for studying the sensitivity of the neutral and ionized atmosphere to solar forcing [Ware et al., 1996; Kursinski et al., 1997; Rocken et al., 1997; Hajj et al., 2000; Kursinski et al., 2000; Rocken et al., 2000; Wickert et al., 2001; Hajj et al., 2002; Schreiner et al., 2007]. The COSMIC (also named FORMOSAT-3 or FORMOSAT-3/ COSMIC) is a joint project between Taiwan and the United States, and consists of 6 satellites, each with three instruments: GPS radio occultation receiver, tiny ionospheric photometer, and triband beacon. Since its launch on 14 April 2006, more than $1,100,000$ and $1,400,000$ occultations (through 4 June 2008) have been obtained globally for the neutral atmosphere and ionosphere, respectively. Here, we report on the existence of the 27-day solar rotation induced signals in this dataset in the ionosphere. The observed variations of electron density in the ionosphere can provide useful constraints on the short-term variability in models, and thereby greatly

\footnotetext{
${ }^{1}$ Research Center for Environmental Changes, Academia Sinica, Taipei, Taiwan.

${ }^{2}$ Graduate Institute of Astronomy, National Central University, Jhongli City, Taiwan.

${ }^{3}$ Division of Geological and Planetary Sciences, California Institute of Technology, Pasadena, California, USA.
}

Copyright 2008 by the American Geophysical Union. 0094-8276/08/2008GL034433 strengthen our understanding of the response of the Earth's ionosphere to solar forcing.

\section{Data Sources}

[3] COSMIC has started reporting atmospheric and ionospheric profiles since 21 April 2006. In the data analysis presented below, we focus on the data collected after day 250 of the year (7 Sept 2006). This is sufficient for the purpose of studying 27-day (and 13.5-day and 54-day) solar signals in the ionosphere, as this gives more than 20 cycles for meaningful statistical analysis. The peak-to-peak amplitude of the signal of interest in this paper exceeds $10^{4}$ electrons $\mathrm{cm}^{-3}$, far above the root-mean-square difference between two collocated COSMIC GPS occultations [Schreiner et al., 2007]; the absolute noise level for electron densities derived from a single occultation remains undetermined. To increase the signal-to-noise ratio for the data, the electron density are daily and zonally (longitudinally) averaged in 36 latitude bins from pole to pole and 51 uniform bins (each with $10 \mathrm{~km}$ ) from the surface to an altitude of $510 \mathrm{~km}$.

[4] To retrieve the 27-day (13.5-day) signal, three spectral filtering techniques are explored. The first technique is the typical fast Fourier transform (FFT). This spectral filter is applied as a convolution of a step function with a Hanning window chosen to obtain a signal from periods above 20-25 (9-10) days and below 35-40 (15-16) days. The period range in each edge of the Hanning window refers to the transition region where frequencies are damped. The filtered signals are not sensitive to the width of the Hanning window used for the Fourier filtering as long as the window is wide enough to cover the signal of interest but not to overlap with strong signals nearby. This is selected to be our reference filter. The second, known as empirical mode decomposition (EMD) [Huang et al., 1998; Wu and Huang, 2004; Wu et al., 2007], is a more sophisticated way of extracting the solar cycle signals. The EMD is designed to take into account all nonlinear and nonstationary features of signals, and decomposes the data into a series of empirical modes. Each mode has a symmetric envelope defined by the local maxima and minima so that its amplitude mean is zero everywhere. The 27-day signal is mainly in EMD mode 4 and a 13.5-day (half of the 27-day period) signal is mainly in mode 3 . The third technique is the Morlet wavelet transform [e.g., Meyers et al., 1993; Torrence and Compo, 1998]. Combination of these three provides tests of the robustness of the retrieved signals. For example, the EMD and a running average are used to remove low frequency signals (periods greater than $\sim 50$ days for 13.5 -day and 27-day signals), followed by the wavelet or Fourier analysis. 


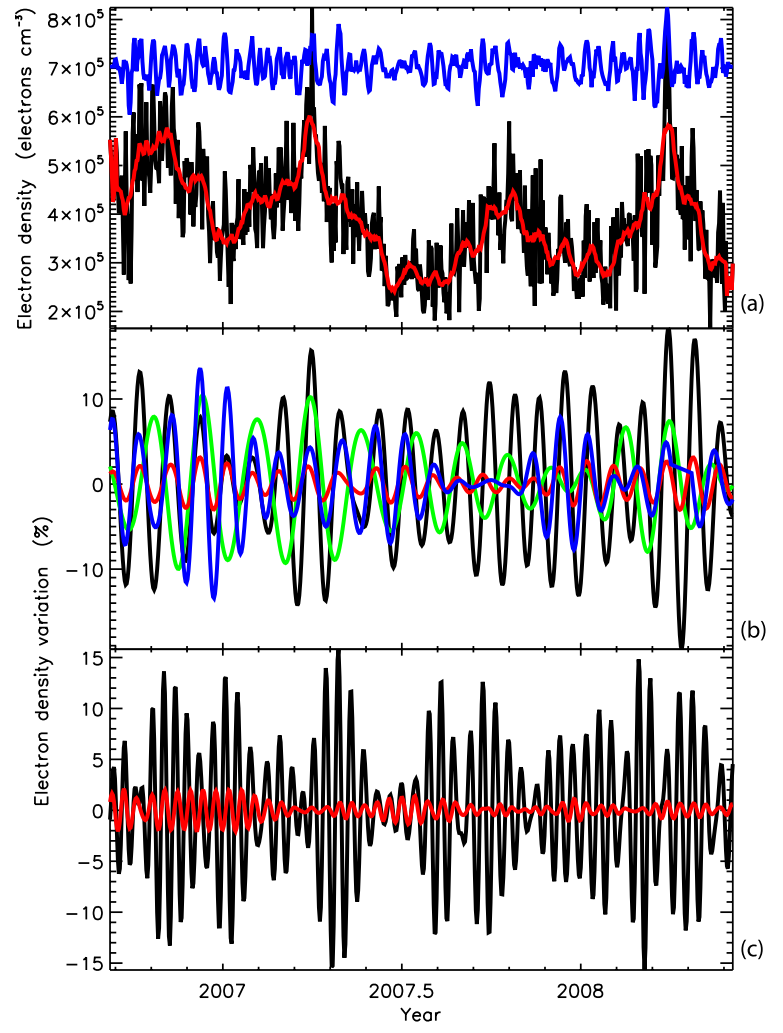

Figure 1. (a) Time series of the observed electron density at $2.5^{\circ} \mathrm{N}$ and $355 \mathrm{~km}$. The red and blue curves show the 15-day running average of the daily data (black) and 5-day running average of the residual between the black and red curves (a constant offset has been applied), respectively. (b) The 27-day (black) and 54-day (green) filtered time series, obtained by applying the FFT filters to the black curve in Figure 1a. The 27-day FFT filtered $10.7-\mathrm{cm}$ and Lyman- $\alpha$ solar indices are shown by the blue and red curves, respectively. The three time series are normalized by their mean values, which are then removed. (c) The FFT filtered 13.5-day time series of the black curve in Figure 1a. The red curve represents the 13.5-day filtered Lyman- $\alpha$ flux.

[5] Proxies of the 27-day solar forcing are solar Lyman- $\alpha$, MgII, and 10.7-cm radio fluxes, and the number of sunspots. Over UV ( $100-300 \mathrm{~nm})$ wavelengths, the amplitude of the solar variability is higher at Lyman- $\alpha$ (see Figure 1) than that at the MgII line ( $\sim 1 \%$ for 27-day cycle) [Snow et al., 2005; Woods et al., 2006]. SORCE/SOLSTICE, started since 2003, consists of daily solar spectra at wavelengths between 116-310 $\mathrm{nm}$ with 1-nm resolution [Sparn et al., 2005; Rottman et al., 2006]. A 5-day running average was applied to the data to reduce high frequency signals that are far away from the signals of interest. In the ionosphere below (above) the F-peak, the electron density variation was shown to be better represented by the $10.7-\mathrm{cm}$ solar flux (sunspots) [Bilitza, 2001], and this 10.7-cm solar index [Pap et al., 1990; Bouwer, 1992] is chosen for the investigation of solar cycle response in the ionosphere. The power spectrum of the solar index ranges from periods of 20 to 40 days, centered at $\sim 27$ days. For consistency, the same filtering methods as those used by the electron data are applied to the solar indices. The 27day filtered time series of Lyman- $\alpha$ and $10.7-\mathrm{cm}$ radio fluxes are shown in Figure 1. The 13.5-day is present in Lyman- $\alpha$ but not in 10.7-cm index. Therefore, Lyman- $\alpha$ is used for the 13.5-day solar cycle signal comparison. See Pap et al. [1990] for a decent summary and review of the solar cycle signals.

\section{Results}

[6] All three filtering methods give consistent results (in both amplitude and phase). So the traditional filtering method, the FFT (technique 1), is chosen for the following discussion. Figure 1 demonstrates an example of the solar cycle signals retrieved from the COSMIC data in the ionosphere. Figure 1a shows the electron density derived at $2.5^{\circ} \mathrm{N}$ and $355 \mathrm{~km}$. To demonstrate the presence of the 13.5 and 27-day signals, we first apply running average to the daily data, followed by a full FFT analysis. The 15-day running average of the daily data (black) is given by the red curve. The 27-day period signals are clearly present by comparing the peak positions of the red curve with the FFT filtered time series in Figure 1b. The blue curve is obtained by applying a 5-day running average to the residual between the black curve and the red curve. The 13.5-day signals are also seen. To better isolate/retrieve the signals of interest, we apply the aforementioned Fourier filters to the daily data. The peak-to-peak amplitude variation of the 27 -day signal in the ionospheric electron density is $\sim 10$ $25 \%$ relative to the mean electron density at this location, or about $2-10 \times 10^{4}$ electrons $\mathrm{cm}^{-3}$. The 27-day filtered $10.7-\mathrm{cm}$ solar flux is shown by the blue curve in Figure 1b. The correlation coefficient for the two is 0.6. The 13.5-day filtered signals are presented in Figure 1c.

[7] The power spectrum of the daily electron data and the confidence level test are presented in Figure 2. The Fourier spectra are strongly affected by the length of the time series

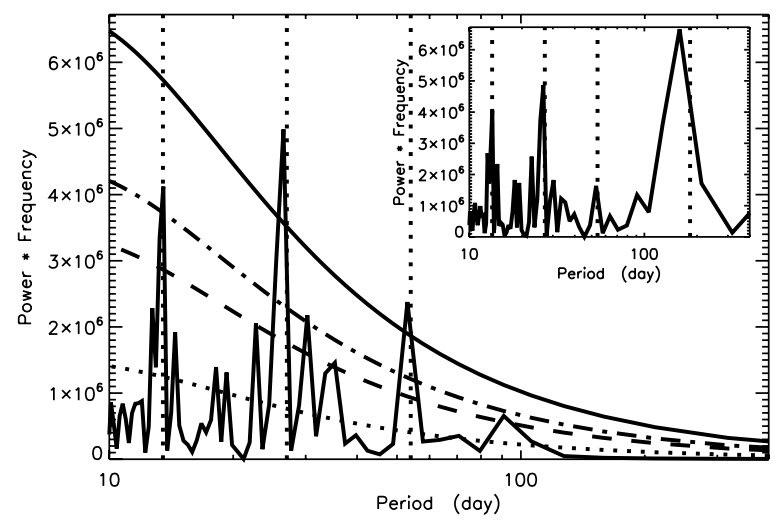

Figure 2. Fourier spectra of the daily electron data (black curve in Figure 1a). The vertical dotted lines mark the periods of $13.5,27,54$, and 182.5 days. The red noise spectrum (dotted) and 90\% (dashed), 95\% (dash-dotted), and 99\% (solid) confidence intervals are also shown. It is obvious that the chance of the signals of interest, especially the 27 day signal, generated by random processes is way below $1 \%$. Signals with periods greater than 100 days have been taken out for statistical analysis. See text for details. Inset: Fourier spectrum of the unfiltered data. 

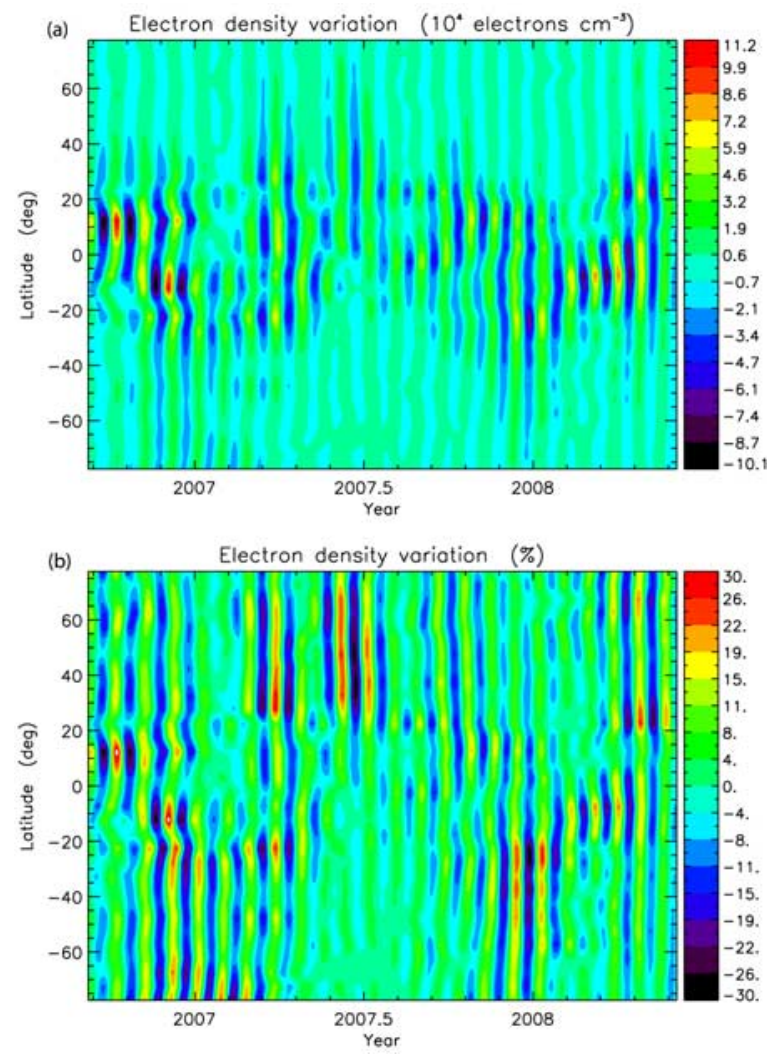

Figure 3. Electron density fluctuation associated with the 27-day cycle at $355 \mathrm{~km}$ : (a) absolute (in $10^{4}$ electrons $\mathrm{cm}^{-3}$ ) and (b) fractional variations (\%).

chosen for the analysis. The filtered time series of the signals of interest from the COSMIC data are above the $95 \%$ confidence level and are less affected by the length of the data (but need to be long enough for statistical meaningful analysis, e.g., at least 10 cycles). The confidence level is defined by the deviation from the red noise (dotted curve) obtained by randomizing high frequency (periods less than $\sim 100$ days) signals in the COSMIC data, because the power is dominated by the semi-annual oscillation and certainly cannot be attributed to noise.

[8] In addition to the known 13.5-day and 27-day solar cycles, a peak of 54-day in the power spectrum plot is also observed. The associated filtered signal is shown by the green curve in Figure 1; the selected Hanning window for the Fourier filtering is 35-40 days and 60-65 days (corresponding to EMD mode 5). Further statistical tests using the EMD technique [Wu and Huang, 2004] verify the significance of the signals. The confidence levels of the modes $3-5(13.5,27$, and 54 days $)$ are $>99 \%$. The two methods (FFT and EMD) of defining noise are similar, but the latter may be more robust. The former is based on the statistics of signals with periods $<100$ days and the latter is from the EMD mode 1, which contains only signals with periods less than 10 days.

[9] Figure 3 shows the FFT filtered time series of electron density at $355 \mathrm{~km}$ (F2 region), where it peaks; the cross sections at other altitudes have similar latitude variation. The spatial pattern of the absolute density variation (Figure 3a) follows the seasonal cycle, i.e., moving to higher latitudes in the summer season. The electron density exhibits maximum amplitude at low latitudes, suggesting that the solar activity is the primarily source of the 27-day cycles observed in the ionosphere [e.g., Rich et al., 2003; Fröhlich and Lean, 2004]. The argument is supported by the good phase coherence between the time series of electron density anomaly and the solar flux index shown in Figure 1. Unlike the absolute variation, the fractional variation (Figure 3b) appears relatively uniform across latitudes but tends to be more variable at higher latitudes of the summer hemisphere. Figure 4 shows the vertical profiles at the equator. The maximum density variation appears at $\sim 400 \mathrm{~km}$ where the electron density peaks, and the fractional variation is relatively uniform across the entire region.

\section{Discussion}

[10] All of the retrieved solar signals from the COSMIC ionospheric data show high spatial coherence, further verifying the existence of the solar cycle signals and the robustness of the retrieval methods described above. A several-day phase difference between latitudes and altitudes is also observed, and we believe the phase is due to ionospheric chemistry and dynamics. The major ion is $\mathrm{O}^{+}$, which has a lifetime due to recombination $\sim 10$ days at the equator at $355 \mathrm{~km}$. Short-period (a few days) wave activities are also observed in the ionosphere [e.g., Liu et al., 2004; Riggin et al., 2006; Liu et al., 2007]. Since the two time
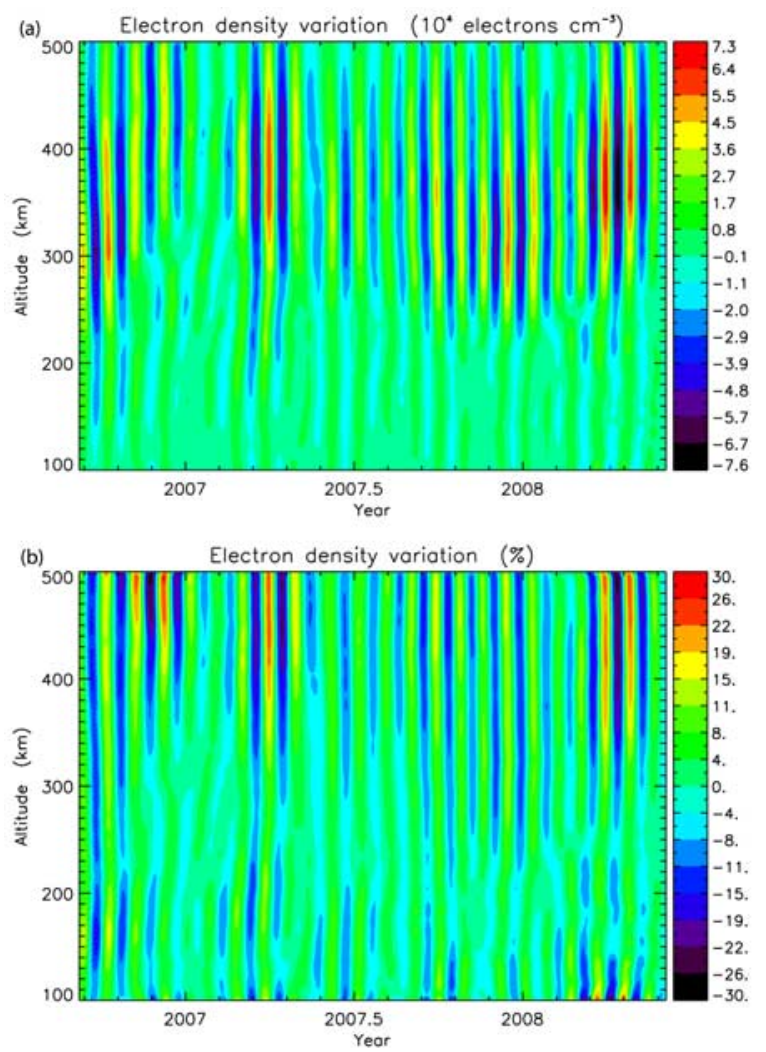

Figure 4. Electron density fluctuation associated with the 27-day cycle at $2.5^{\circ} \mathrm{N}$ : (a) absolute (in $10^{4}$ electrons $\mathrm{cm}^{-3}$ ) and (b) fractional variations (\%). 
constants are not small compared with the 27-day solar cycle, some phase incoherence is expected.

[11] The amplitudes of 13.5-day and 54-day signals are similar to that at 27-day. The 13.5-day signal may be attributed to solar forcing (see Figure 1c). The 54-day signal may be explained by two mechanisms. First, the signal is related to the 51-day solar cycle [Pap et al., 1990]. Second, it may be a subharmonic response of the atmosphere to the 27-day solar forcing. The period doubling phenomenon is known to occur in nonlinear systems [see, e.g., Marts et al., 2007] and in the upper atmosphere. Notable examples include the 2-day oscillation in $\mathrm{O}_{3}$, temperature and water vapor observed in the mesosphere [Azeem et al., 2001; Limpasuvan and $W u, 2003]$. In these cases the solar forcing is at 1-day, but a nonlinear response of the atmosphere could result in a 2-day oscillation, as demonstrated in a simple but realistic model of the upper atmosphere [Sonnemann and Grygalashvyly, 2005].

[12] COSMIC offers a great opportunity for studying the Earth ionosphere's response to solar forcing. We note at present the Sun is near the minimum of the 11-year cycle. We expect that the variation could be larger when the Sun reaches solar maximum in a few years. Moreover, the peakto-peak 11-year variation at Lyman- $\alpha$ can be as large as $50 \%$, an order of magnitude greater than the 27-day (and 13.5 -day) variation ( $\sim 5 \%$ ) of Lyman- $\alpha$ at present. Thus, the GPS data will not only provide a means to understand the short-term variability in the ionosphere such as 13.5-day and 27-day cycles mentioned here, but also long-term (e.g., decadal and centennial) variations.

[13] Acknowledgments. We thank S. Sander, K. K. Tung, and D. Waliser for valuable inputs. This work was supported in part by an NSC grant 96-2628-M-001-018 to the Academia Sinica. KFL, RLS and YLY were supported in part by NASA MAP program via JPL grant P480501 to the California Institute of Technology. The authors acknowledge the NSPO and UCAR CDAAC groups for providing FORMOSAT-3/COSMIC GPS radio occultation data.

\section{References}

Azeem, S. M. I., S. E. Palo, D. L. Wu, and L. Froidevaux (2001), Observations of the 2-day wave in UARS MLS temperature and ozone measurements, Geophys. Res. Lett., 28, 3147-3150.

Bilitza, D. (2001), International reference ionosphere 2000, Radio Science, $36,261-275$.

Bouwer, S. D. (1992), Periodicities of solar irradiance and solar-activity indexes, II, Sol. Phys., 142, 365-389.

Fröhlich, C., and J. Lean (2004), Solar radiative output and its variability: Evidence and mechanisms, Astron. Astrophys. Rev., 12, 273-320.

Hajj, G. A., L. C. Lee, X. Q. Pi, L. J. Romans, W. S. Schreiner, P. R. Straus, and C. M. Wang (2000), COSMIC GPS ionospheric sensing and space weather, Terr. Atmos. Oceanic Sci., 11, 235-272.

Hajj, G. A., E. R. Kursinski, L. J. Romans, W. I. Bertiger, and S. S. Leroy (2002), A technical description of atmospheric sounding by GPS occultation, J. Atmos. Sol. Terr. Phys., 64, 451-469.

Huang, N. E., et al. (1998), The empirical mode decomposition and the Hilbert spectrum for nonlinear and non-stationary time series analysis, Proc. R. Soc. London, Ser. A, 454, 903-995.

Kursinski, E. R., G. A. Hajj, J. T. Schofield, R. P. Linfield, and K. R. Hardy (1997), Observing Earth's atmosphere with radio occultation measurements using the Global Positioning System, J. Geophys. Res., 102, $23,429-23,465$
Kursinski, E. R., G. A. Hajj, S. S. Leroy, and B. Herman (2000), The GPS radio occultation technique, Terr. Atmos. Oceanic Sci., 11, 53-114.

Limpasuvan, V., and D. L. Wu (2003), Two-day wave observations of UARS Microwave Limb Sounder mesospheric water vapor and temperature, J. Geophys. Res., 108(D10), 4307, doi:10.1029/2002JD002903.

Liu, H.-L., E. R. Talaat, R. G. Roble, R. S. Lieberman, D. M. Riggin, and J.-H. Yee (2004), The 6.5-day wave and its seasonal variability in the middle and upper atmosphere, J. Geophys. Res., 109, D21112, doi:10.1029/2004JD004795.

Liu, H.-L., T. Li, C.-Y. She, J. Oberheide, Q. Wu, M. E. Hagan, J. Xu, R. G. Roble, M. G. Mlynczak, and J. M. Russell III (2007), Comparative study of short-term diurnal tidal variability, J. Geophys. Res., 112, D18108, doi:10.1029/2007JD008542.

Marts, B., et al. (2007), Period doubling in a periodically forced Belousov-Zhabotinsky reaction, Phys. Rev. E, 76(2), 026213, doi:10.1103/ PhysRevE.73.026213.

Meyers, S. D., B. G. Kelly, and J. J. Obrien (1993), An introduction to wavelet analysis in oceanography and meteorology-with application to the dispersion of Yanai waves, Mon. Weather Rev., 121, 2858-2866.

Pap, J., et al. (1990), Periodicities of solar irradiance and solar-activity indexes, I, Sol. Phys., 129, 165-189.

Rich, F. J., P. J. Sultan, and W. J. Burke (2003), The 27-day variations of plasma densities and temperatures in the topside ionosphere, J. Geophys. Res., 108(A7), 1297, doi:10.1029/2002JA009731.

Riggin, D. M., et al. (2006), Observations of the 5-day wave in the mesosphere and lower thermosphere, J. Atmos. Sol. Terr. Phys., 68, 323-339.

Rocken, C., et al. (1997), Analysis and validation of GPS/MET data in the neutral atmosphere, J. Geophys. Res., 102, 29,849-29,866.

Rocken, C., Y. H. Kuo, W. S. Schreiner, D. Hunt, S. Sokolovskiy, and C. McCormick (2000), COSMIC system description, Terr. Atmos. Oceanic Sci., 11, 21-52.

Rottman, G. J., T. N. Woods, and W. McClintock (2006), SORCE solar UV irradiance results, Adv. Space Res., 37, 201-208, doi:10.1016/ j.asr.2005.02.072.

Schreiner, W., C. Rocken, S. Sokolovskiy, S. Syndergaard, and D. Hunt (2007), Estimates of the precision of GPS radio occultations from the COSMIC/FORMOSAT-3 mission, Geophys. Res. Lett., 34, L04808, doi:10.1029/2006GL027557.

Snow, M., W. E. McClintock, T. N. Woods, O. R. White, J. W. Harder, and G. Rottman (2005), The MgII index from SORCE, Sol. Phys., 230, 325-344.

Sonnemann, G. R., and M. Grygalashvyly (2005), On the two-day oscillations and the day-to-day variability in global 3-D-modeling of the chemical system of the upper mesosphere/mesopause region, Nonlinear Processes Geophys., 12, 691-705.

Sparn, T. P., G. Rottman, T. N. Woods, B. D. Boyle, R. Kohnert, S. Ryan, R. Davis, R. Fulton, and W. Ochs (2005), The SORCE spacecraft and operations, Sol. Phys., 230, 71-89.

Torrence, C., and G. P. Compo (1998), A practical guide to wavelet analysis, Bull. Am. Meteorol. Soc., 79, 61-78.

Ware, R., et al. (1996), GPS sounding of the atmosphere from low Earth orbit: Preliminary results, Bull. Am. Meteorol. Soc., 77, 19-40.

Wickert, J., et al. (2001), Atmosphere sounding by GPS radio occultation: First results from CHAMP, Geophys. Res. Lett., 28, 3263-3266.

Woods, T., J. Harder, G. Kopp, B. McClintock, P. Pilewskie, M. Snow, and V. George (2006), Solar irradiance variability during the SORCE mission, Earth Obs., 18, 8-14.

Wu, Z. H., and N. E. Huang (2004), A study of the characteristics of white noise using the empirical mode decomposition method, Proc. R. Soc. London, Ser. A, 460, 1597-1611.

$\mathrm{Wu}, \mathrm{Z}$. H., et al. (2007), On the trend, detrending, and variability of nonlinear and nonstationary time series, Proc. Natl. Acad. Sci. U. S. A., 104, $14,889-14,894$

K.-F. Li, R.-L. Shia, and Y. L. Yung, Division of Geological and Planetary Sciences, California Institute of Technology, Pasadena, CA 91125, USA.

M.-C. Liang, Research Center for Environmental Changes, Academia Sinica, Taipei 115, Taiwan. (mcl@rcec.sinica.edu.tw) 